\title{
Dermatomyositis Flare With Immune Checkpoint Inhibitor Therapy for Melanoma
}

\author{
Rachel Thomas ${ }^{1}$, Hamish Patel ${ }^{2}$, Joshua Scott ${ }^{3}$ \\ 1. Internal Medicine, Wright State University Boonshoft School of Medicine, Dayton, USA 2. Rheumatology, Brooke \\ Army Medical Center, Fort Sam Houston, USA 3. Rheumatology, Wright-Patterson Medical Center, Dayton, USA
}

Corresponding author: Rachel Thomas, rachel.thomas488@gmail.com

\begin{abstract}
Immune checkpoint inhibitor (ICI) medications have seen expanded use in the management of numerous malignancies. These therapies encompass a unique spectrum of immune-related adverse events (irAEs). Rheumatological irAEs from ICIs have been described in various case reports; however, limited literature exists regarding the treatment of patients with pre-existing myositis. We describe a case of myositis flare after initiation of nivolumab for metastatic melanoma in a patient who had previously achieved remission of dermatomyositis.
\end{abstract}

Categories: Dermatology, Oncology, Rheumatology

Keywords: myositis, immune checkpoint inhibitors (icis), immune-related adverse effects, metastatic melanoma, nivolumab, myositis flare, dermatomyositis

\section{Introduction}

Immune checkpoint inhibitors (ICIs) have seen expanded use in the management of numerous malignancies. These medications were first approved for use in metastatic melanoma and are now approved for use in other malignancies including but not limited to non-small cell lung cancers, urothelial cancers, gastric adenocarcinoma, and Hodgkin's lymphoma [1]. While these therapies have provided significant survival benefit, they encompass a unique spectrum of immune-related adverse events (irAEs). The most common irAEs are dermatologic and gastrointestinal in nature; however, endocrine, hepatic, pulmonary, and neurologic side effects have also been reported [1-4]. Rheumatological irAEs from ICIs have been described in various case reports. However, limited literature exists on the use of ICIs in patients with pre-existing dermatomyositis, and completion of ICI therapy after a myositis flare is uncommon. Here we describe a case of flare of dermatomyositis after initiation of nivolumab for metastatic melanoma in a patient who had previously achieved myositis remission on rituximab.

Review began 03/16/2021 Review ended 04/07/2021 Published 04/09/2021

(c) Copyright 2021

Thomas et al. This is an open access article distributed under the terms of the Creative Commons Attribution License CC-BY 4.0., which permits unrestricted use, distribution, and reproduction in any medium, provided the original author and source are credited.

\section{Case Presentation}

A 36-year-old female presented to the rheumatology clinic with progressive weakness and rash during her second pregnancy. The patient was diagnosed with dermatomyositis based on decreased proximal muscle strength (3/5) in the bilateral lower extremities, Holster sign rash, Gottron papules, elevated creatine kinase (CK) (564 U/L), elevated aldolase (11.8 U/L), elevated inflammatory markers, high antinuclear antibody titer $(>1: 1280)$, and positive transcriptional intermediary factor 1 -gamma (TIF1- $\gamma)$. Cancer screening including mammography, breast ultrasound, transvaginal ultrasound, pelvic MRI, and skin examination was negative for evidence of malignancy. She was subsequently treated with 5-20 mg of prednisone daily during her pregnancy. However, post-partum, the patient had a recurrence of her myositis symptoms with corresponding changes on her MRI of the bilateral lower extremities. Treatment with prednisone and mycophenolate mofetil failed to control her disease. Rituximab was initiated for further steroid-sparing. Initial rituximab dosing was two 1-gram infusions 14 days apart. Rituximab was continued for the maintenance of remission dosed at $500 \mathrm{mg}$ infusions separated by 14 days every six months. Four months after her second maintenance dose of rituximab, the patient was diagnosed with stage IIIA melanoma after biopsy of an evolving pigmented lesion on her left thigh. The patient underwent wide local excision and sentinel node dissection. Two out of three nodes were positive for micro-metastasis; however, positron emission tomography (PET) was negative for distant metastasis. The patient elected to undergo adjuvant treatment with nivolumab. After the second infusion, the patient experienced recurrent myositis symptoms including severe fatigue, proximal weakness, Gottron papules, and elevated CK (1,071 U/L). MRI of the proximal bilateral lower extremities again demonstrated inflammatory changes, consistent with her prior dermatomyositis episodes (Figure 1). The patient was treated per the American Society of Clinical Oncology guidelines with $20 \mathrm{mg}$ of prednisone tapered over six months [1]. She was able to complete the full 12 months of nivolumab therapy without any further steroids or flares of her dermatomyositis. 


\title{
Cureus
}
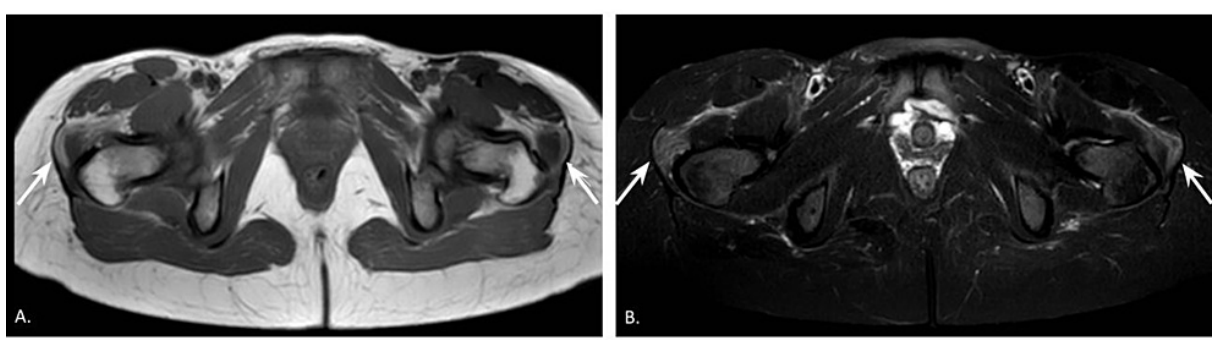

\author{
FIGURE 1: MRI axial T1 (A) and T2 (B) images of the bilateral lower \\ extremities during dermatomyositis flare on nivolumab therapy. Muscle \\ edema in the bilateral gluteus minimus and gluteus medius muscles \\ was noted.
}

\section{Discussion}

ICIs block specific pathways in the immune checkpoint cycle, resulting in increased T-cell activation. Proteins typically expressed on T-cells known as cytotoxic T-lymphocyte-associated-4 (CTLA4) and programmed cell death protein 1 (PD-1) bind with their respective ligands, CD80/CD86 and programmed cell death ligand 1 (PD-L1), causing T-cell deactivation. Cancer cells mimic these ligands in order to evade and dampen the natural immune response. The use of ICIs, by blocking either CTLA4, PD-1, or PD-L1, results in an upregulation of the immune response, which helps target and destroy tumor cells [5]. However, due to non-specific T-cell stimulation, these therapies result in various immune-related adverse events ranging from mild to severe.

ICI use among individuals with underlying autoimmune conditions can be limited because of not only their side effect profile but also the ability to dampen the aforementioned T-cell regulation. While it is true that most irAEs manifest as dermatologic or gastrointestinal issues, emerging literature and case reports have noted a growing number of rheumatologic irAEs [6-7]. The most common of these symptoms include primary arthritis, sicca symptoms, and polymyalgia rheumatica-like syndromes [4]. A retrospective study by Abdel-Wahab et al. noted that $75 \%$ of patients with a preexisting autoimmune disease developed an irAE with ICI treatment [8]. More than half of these patients experienced a flare of their prior disorder, whereas less than one-third developed a different irAE. In a more recent study by the Dutch Melanoma Treatment Registry, the rate of irAE was 30\% irrespective of the presence of an underlying autoimmune condition [9].

Myositis is a rare irAE of ICI therapy, with few cases reported. A review of prior case series showed the development of myositis in a few patients without an underlying autoimmune condition [10-11]. The greater concern lies with the severe or fatal complications of myositis. Progression of the inflammatory myopathy can result in end organ damage, with the most severe causing myocarditis. One particular literature review from Sweden revealed that 29 of 180 cases of myositis were complicated by myocarditis, leading to a significantly increased mortality rate (51.7\% versus $14.9 \%$ in ICI-related myositis without myocarditis) [12].

\section{Conclusions}

ICI therapy is associated with a unique spectrum of adverse events due to inhibition of the usual T-cell regulatory cycle. For those with an underlying autoimmune condition, the increased T-cell activation secondary to ICI commonly triggers a disease flare. While most rheumatological irAEs can be managed with corticosteroid therapy, the risks and benefits of initiating or continuing ICI therapy must be carefully weighed. ICI therapy is commonplace in oncology, and understanding the spectrum of irAEs is paramount in management. The case in this report not only highlights the ability to successfully complete ICI therapy in a patient with preexisting dermatomyositis but also illustrates the importance of recognizing and treating irAEs early as complications can be swift and severe.

\section{Additional Information}




\section{Disclosures}

Human subjects: Consent was obtained or waived by all participants in this study. Conflicts of interest: In compliance with the ICMJE uniform disclosure form, all authors declare the following: Payment/services info: All authors have declared that no financial support was received from any organization for the submitted work. Financial relationships: All authors have declared that they have no financial relationships at present or within the previous three years with any organizations that might have an interest in the submitted work. Other relationships: All authors have declared that there are no other relationships or activities that could appear to have influenced the submitted work.

\section{References}

1. Brahmer JR, Lacchetti C, Schneider BJ, et al.: Management of immune-related adverse events in patients treated with immune checkpoint inhibitor therapy: American Society of Clinical Oncology Clinical Practice Guideline. J Clin Oncol. 2018, 36:1714-68. 10.1200/JCO.2017.77.6385

2. Sheik Ali S, Goddard AL, Luke JJ, Donahue H, Todd DJ, Werchniak A, Vleugels RA: Drug-associated dermatomyositis following ipilimumab therapy: a novel immune-mediated adverse event associated with cytotoxic T-lymphocyte antigen 4 blockade. JAMA Dermatol. 2015, 151:195-9. 10.1001/jamadermatol.2014.2233

3. Bourgeois-Vionnet J, Joubert B, Bernard E, et al.: Nivolumab-induced myositis: a case report and a literature review. J Neurol Sci. 2018, 387:51-3. 10.1016/j.jns.2018.01.030

4. Shah M, Tayar JH, Abdel-Wahab N, Suarez-Almazor ME: Myositis as an adverse event of immune checkpoint blockade for cancer therapy. Semin Arthritis Rheum. 2019, 48:736-40. 10.1016/j.semarthrit.2018.05.006

5. Maul LV, Weichenthal M, Kähler KC, Hauschild A: Successful anti-PD-1 antibody treatment in a metastatic melanoma patient with known severe autoimmune disease. J Immunother. 2016, 39:188-90. 10.1097/CJI.0000000000000118

6. Lidar M, Giat E, Garelick D, et al.: Rheumatic manifestations among cancer patients treated with immune checkpoint inhibitors. Autoimmun Rev. 2018, 17:284-9. 10.1016/j.autrev.2018.01.003

7. Richter MD, Crowson C, Kottschade LA, Finnes HD, Markovic SN, Thanarajasingam U: Rheumatic syndromes associated with immune checkpoint inhibitors: a single-center cohort of sixty-one patients. Arthritis Rheumatol. 2019, 71:468-75. 10.1002/art.40745

8. Abdel-Wahab N, Shah M, Lopez-Olivo MA, Suarez-Almazor ME: Use of immune checkpoint inhibitors in the treatment of patients with cancer and preexisting autoimmune disease: a systematic review. Ann Intern Med. 2018, 168:121-30. 10.7326/M17-2073

9. van der Kooij MK, Suijkerbuijk KPM, Aarts MJB, et al.: Safety and efficacy of checkpoint inhibition in patients with melanoma and preexisting autoimmune disease: a cohort study [Online ahead of print]. Ann Intern Med. 2021, 10.7326/M20-3419

10. Calabrese C, Kirchner E, Kontzias A, Velcheti V, Calabrese LH: Rheumatic immune-related adverse events of checkpoint therapy for cancer: case series of a new nosological entity. RMD Open. 2017, 3:000412. 10.1136/rmdopen-2016-000412

11. Narváez J, Juarez-López P, LLuch J, et al.: Rheumatic immune-related adverse events in patients on antiPD-1 inhibitors: fasciitis with myositis syndrome as a new complication of immunotherapy. Autoimmun Rev. 2018, 17:1040-5. 10.1016/j.autrev.2018.05.002

12. Anquetil C, Salem JE, Lebrun-Vignes B, et al.: Immune checkpoint inhibitor-associated myositis: expanding the spectrum of cardiac complications of the immunotherapy revolution. Circulation. 2018, 138:743-5. 10.1161/CIRCULATIONAHA.118.035898 